\title{
Male mandible trimorphism in the stag beetle Dorcus rectus (Coleoptera: Lucanidae)
}

\author{
YUTAKA IGUCHI \\ Laboratory of Biology, Yamashita-cho 1-10-6, Okaya City, Nagano Prefecture, 394-0005, Japan; e-mail: bio-igu@f8.dion.ne.jp
}

Key words. Lucanidae, Dorcus rectus, stag beetle, mandible, trimorphism, scaling relationship, discontinuous model

\begin{abstract}
Males in many beetles show horn or mandible dimorphism. The stag beetle Dorcus rectus was assumed to have dimorphic males, but in this species there are not two but three visually distinct mandibular phenotypes. The aim of this study is to determine if the three phenotypes represent an allometric trimorphism in this species by studying the scaling relationship between body length and mandible length in 148 adult males and 31 adult females. The relationship was fitted using the following four statistical models; a simple line, a logistic curve, a continuous segmented line and a discontinuous line. The best-fitting model for the males was a discontinuous line with two break points and that for the females a simple line. The male data was also well fitted by a logistic curve. The discontinuous linear model divided the males into three groups that were in good agreement with the three phenotypes. These results suggest that a subtle allometric trimorphism possibly exists in males of this species. Small males of this species have no distinct teeth on their mandibles, whereas small males of D. striatipennis and D. curvidens, which are closely related to D. rectus, have a pair of distinct teeth on their mandibles. The phylogenetic relationship of these Dorcus species suggests that the trimorphism in $D$. rectus may have arisen through the loss of teeth in small males.
\end{abstract}

\section{INTRODUCTION}

Males in many beetle species show great variation in the expression of secondary sexual traits such as horns or mandibles. Such variation in males is often discontinuous and males are either large or small with respect to their horn or mandible size (Inukai, 1924; Clark, 1977; Eberhard, 1982; Goldsmith, 1985; Cook, 1987; Rasmussen, 1994; Siva-Jothy, 1987; Eberhard \& Gutierrez, 1991; Kawano, 1995, 1997; Iguchi, 1998; Emlen \& Nijhout, 2000; Moczek \& Emlen, 2000; Shiokawa \& Iwahashi, 2000; Tatsuta et al., 2001, 2004). Recently, however, Rowland \& Emlen (2009) indicated that several families of the Coleoptera (e.g., Scarabaeidae and Lucanidae) considered to be male dimorphic actually contain species that are trimorphic in terms of their horns or mandibles. Nevertheless, there are very few reports of such trimorphism in beetles (but see Eberhard et al., 2000; Iguchi, 2002).

Males of Dorcus rectus (Motschulsky) are considered to be dimorphic with respect to mandible length (Iguchi, 2001a). However, they also show three mandible types with respect to the number of teeth (Fig. 1a-c; Kurosawa et al., 1988; Iguchi, 1992), whereas females have only one type of mandible (Fig. 1d). As mandible length increases, the number of teeth seems to increase in males (Iguchi, 1992). However, the relationship between mandible length and mandible shape has not yet been examined in detail. Moreover, there are no morphometric studies on females of this species.

This paper uses the following four models for the scaling relationship between body length and mandible length in D. rectus; a simple line, a logistic curve (Fig. 2a), a continuous segmented line (Fig. 2b) and a discontinuous line (Fig. 2c). These models have been used to determine horn or mandible dimorphism. However, there are very few studies that compare these models in detail (Knell, 2009). In this paper the scaling relationship is determined using these models and the possibility of male allometric trimorphism in D. rectus discussed. Rowland \& Emlen (2009) suggest that trimorphism will be difficult to detect using current methods. However, this paper shows that the comparison of these models, especially the use of the discontinuous model, might help detect a subtle allometric trimorphism. This paper presents the bestfitting models for males and females and then discusses the evolution of their mandibles on the basis of the phylogenetic relationship of the species of Dorcus.

\section{MATERIAL AND METHODS}

\section{Sampling and measurement}

A total of 148 adult males and 31 adult females of $D$. rectus were collected at sap sites on oak trees on 22 days between 20 July and 5 September 2002 in Nirasaki City, Yamanashi Prefecture, Japan. For each living male and female, body length (from the front of the head excluding the mandibles to the tip of the elytra) and mandible length (the greatest length of the left or right mandible measured in a straight line) were measured to the nearest $0.1 \mathrm{~mm}$ with a slide caliper in the laboratory. Voucher specimens are deposited in the Laboratory of Biology, Okaya City, Japan.

\section{Classification of the mandible types}

The mandibles of large males differ in shape from those of small males (Fig. 1). Males can be visually divided into three types, as suggested in previous studies (Kurosawa et al., 1988; Iguchi, 1992); males with two pairs of teeth (Fig. 1a; $n=43$ ), males with one pair (Fig. 1b; $n=75$ ) and males with no teeth on their mandibles (Fig. 1c; $n=30$ ). On the other hand, females only ever have one pair of teeth on their mandibles (Fig. 1d; $n=$ $31)$. 


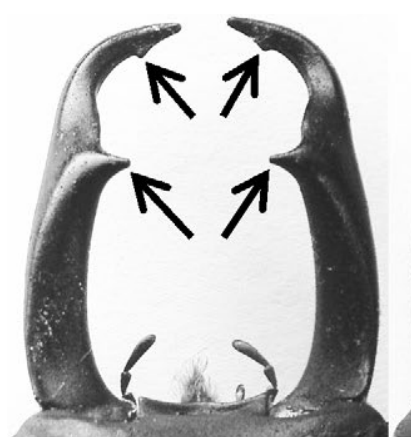

(a)

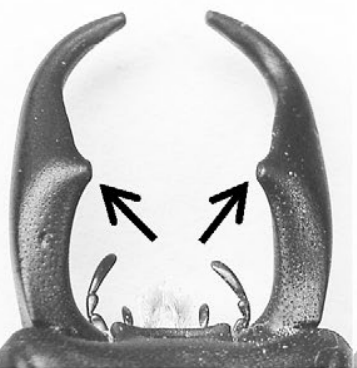

(b)

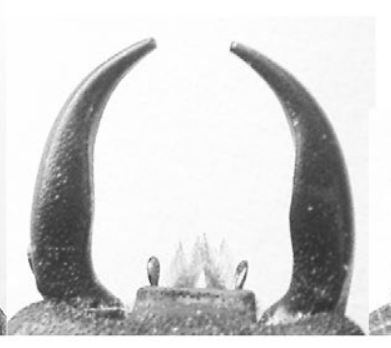

(c)

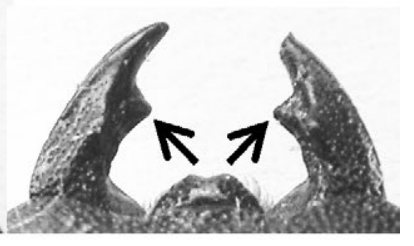

(d)

Fig. 1. Mandibles of Dorcus rectus of different shapes. (a) Male mandibles with two pairs of teeth (arrows). (b) Male mandibles with one pair of teeth (arrows). (c) Male mandibles with no teeth. (d) Female mandibles with one pair of teeth (arrows).

\section{Data analyses}

This study examined the following four statistical models, where $x$ is body length, $y$ is mandible length and $a, b, c$ and $d$ are parameters.

(a) Simple line

$$
y=a x+b
$$

(b) Logistic curve (Fig. 2a)

$$
y=\frac{a}{1+b \exp (-c x)}
$$

The curve is divided into three parts (I, II and III) by two "break" points $\left(B_{1}\right.$ and $\left.B_{2}\right)$, in which the magnitude of the second derivative is largest, i.e. the slope changes fastest.

(c) Continuous segmented line with one or two break points (Fig. 2b)

This model follows Eberhard \& Gutierrez's (1991) model 1. The model with one break point is expressed as

$$
y=a+b x+c\left(x-B_{1}\right) D,
$$

where $D=0$ when $x<B_{1}$ and $D=1$ when $\mathrm{x} \geq B_{1}$. In this model, the slope changes at the break point $x=B_{1}$, but the fitted line is continuous. The model with two break points is defined analogously.

(d) Discontinuous line with one or two break points (Fig. 2c)

This model follows Eberhard \& Gutierrez's (1991) model 2. The model with one break point is expressed as

$$
y=a+b x+c\left(x-B_{1}\right) D+d D,
$$

where $D=0$ when $x<B_{1}$ and $D=1$ when $\mathrm{x} \geq B_{1}$. In this model, the parameter $d$ expresses the magnitude of discontinuity at the break point $x=B_{1}$. Therefore, when $d$ is significantly different from zero, it is concluded that the regression line is discontinuous at $x=B_{1}$. The model with two break points is defined analogously.

The models were fitted by least-squares regression, i.e., by minimizing the sum of squares of residuals (SSR) from the line or curve, in the Maxima and Gnuplot programs (freely available at http://maxima.sourceforge.net and http://www.gnuplot.info).

In the logistic model, the $x$-values of the two "break" points were determined by differentiating the fitted function with respect to $x$. When the two "break" points fell within the range of the data, the relationship between body length and mandible length was called sigmoidal (S-shaped).

In the continuous segmented and discontinuous models, SSR was repeatedly calculated by changing the $x$-values of the break points every $0.01 \mathrm{~mm}$ in the range of the data. In this way, the break points were determined by the smallest SSR. For all the models, the normality of residuals was confirmed by the Shapiro-Wilk test $(\mathrm{P}>0.3)$.

Finally, the corrected Akaike's information criterion (AICc) was calculated for each model as

$$
\mathrm{AICc}=n \ln \left(\frac{\mathrm{SSR}}{n}\right)+\frac{2 k(k+1)}{n-k-1}
$$

where $n$ is the number of data points, $k$ is the number of parameters plus one and $\ln$ is the natural logarithm (Motulsky \& Christopoulos, 2004). AICc compensates for the difference in number of parameters in statistical models and the model with the lowest AICc value is considered to be the best descriptor of the data (Motulsky \& Christopoulos, 2004; Knell, 2009). Models

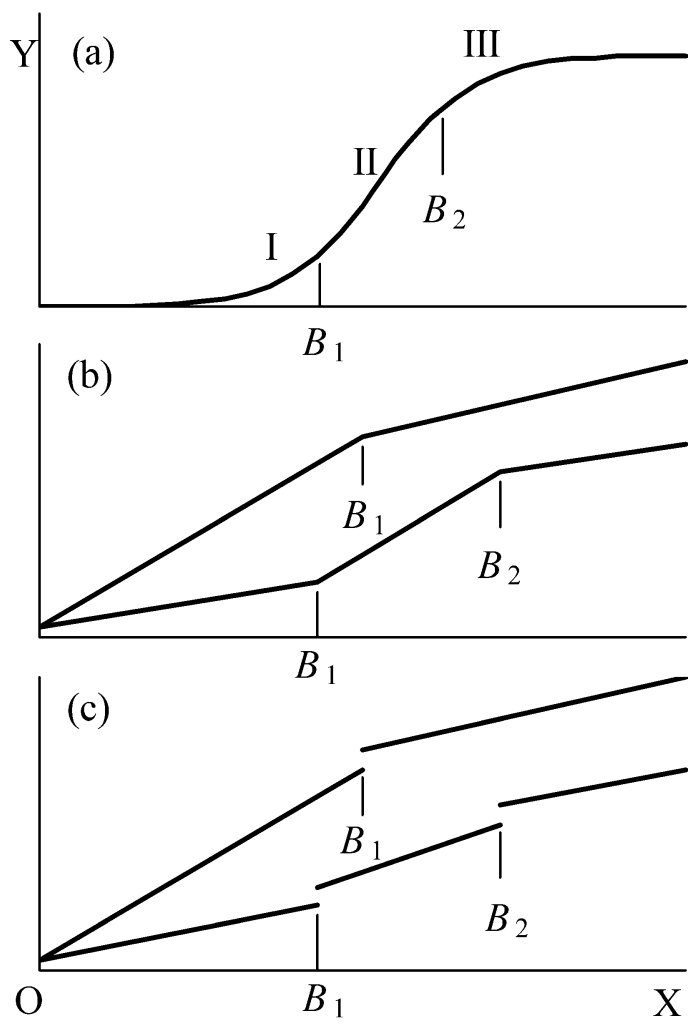

Fig. 2. Statistical models. (a) Logistic curve. This is divided into the three parts (I, II and III) by two break points ( $B_{1}$ and $B_{2}$ ), at which the slope changes fastest. (b) Continuous segmented line with one or two break points. (c) Discontinuous line with one or two break points. 
TABLE 1. Basic statistics of body length and mandible length.

\begin{tabular}{|c|c|c|c|c|c|c|c|}
\hline \multirow{2}{*}{ Sex } & \multirow{2}{*}{$\mathrm{n}$} & \multicolumn{3}{|c|}{ Body length (mm) } & \multicolumn{3}{|c|}{ Mandible length $(\mathrm{mm})$} \\
\hline & & Mean & SD & Range & Mean & SD & Range \\
\hline Male & 148 & 24.1 & 3.2 & $17.8-32.4$ & 7.5 & 2.7 & $2.6-13.3$ \\
\hline Female & 31 & 22.9 & 2.0 & $20.1-27.8$ & 2.4 & 0.3 & $1.9-3.2$ \\
\hline
\end{tabular}

presented in this study differ in the number of parameters. Therefore, AICc was used to choose the best-fitting model.

The evidence ratio is also calculated. It is the relative likelihood of a model being more correct than the best-fitting model (Burnham \& Anderson, 2002). For example, a model with an evidence ratio of 2 is half as likely to be correct as the bestfitting model. In other words, the best-fitting model is twice as strongly supported when the evidence ratio is 2 . Models with evidence ratios $<2.7$ are considered to have substantial support (Burnham \& Anderson, 2002).

Degree of agreement between the classifications of males based on the best-fitting model and the shape of their mandibles

Fleiss' kappa statistic $k$ (Fleiss, 1971, 1981) was used to determine the degree of agreement between the classifications of males using the best-fit model and that based on mandible shape. This statistic $k$ varies between 1 (perfect agreement) and -1 (perfect disagreement), and $k=0$ indicates that the agreement can be explained solely by chance. Generally, $k>0.75$ denotes excellent agreement, $0.40<k<0.75$ fair to good agreement and $k<0.40$ poor agreement (Fleiss, 1981).

\section{RESULTS}

The basic statistics of body length and mandible length are shown in Table 1.

The data for males were fitted best by the discontinuous line with two break points (Fig. 3a and Table 2). The logistic curve model also had substantial support (evidence ratio 2.4) and suggested an S-shaped relationship between body length and mandible length with two "break" points (Fig. 3b). In contrast, the best-fitting

TABLE 2. The sum of squares of residuals (SSR), corrected Akaike's information criterion (AICc) and evidence ratio (ER) for the relationship between body length and mandible length in male and female Dorcus rectus. The ER values were compared with the lowest AICc (in bold).

\begin{tabular}{lcrrr}
\hline Sex & Model & SSR & AICc & \multicolumn{1}{c}{ ER } \\
\hline \multirow{4}{*}{ Male } & Simple line & 37.63 & $-196.52 .2 \cdot 10^{7}$ \\
& Logistic curve & 29.87 & -228.6 & 2.4 \\
& Continuous line with 1 break point & 31.14 & -220.3 & 155.9 \\
& Continuous line with 2 break points & 29.82 & -222.3 & 56.2 \\
& Discontin. line with 1 break point & 29.31 & -227.0 & 5.3 \\
& Discontin. line with 2 break points & 27.39 & $-\mathbf{2 3 0 . 4}$ & 1 \\
\hline \multirow{4}{*}{ Female } & 1.03 & $\mathbf{- 9 8 . 7}$ & 1 \\
& Simple line & 1.01 & -96.5 & 3.0 \\
& Logistic curve & & 1 \\
& Continuous line with 2 break points & 0.89 & -91.2 & 42.0 \\
& Discontin. line with 1 break point & 0.90 & -94.4 & 8.7 \\
& Discontin. line with 2 break points & 0.75 & -88.6 & 152.8 \\
\hline
\end{tabular}
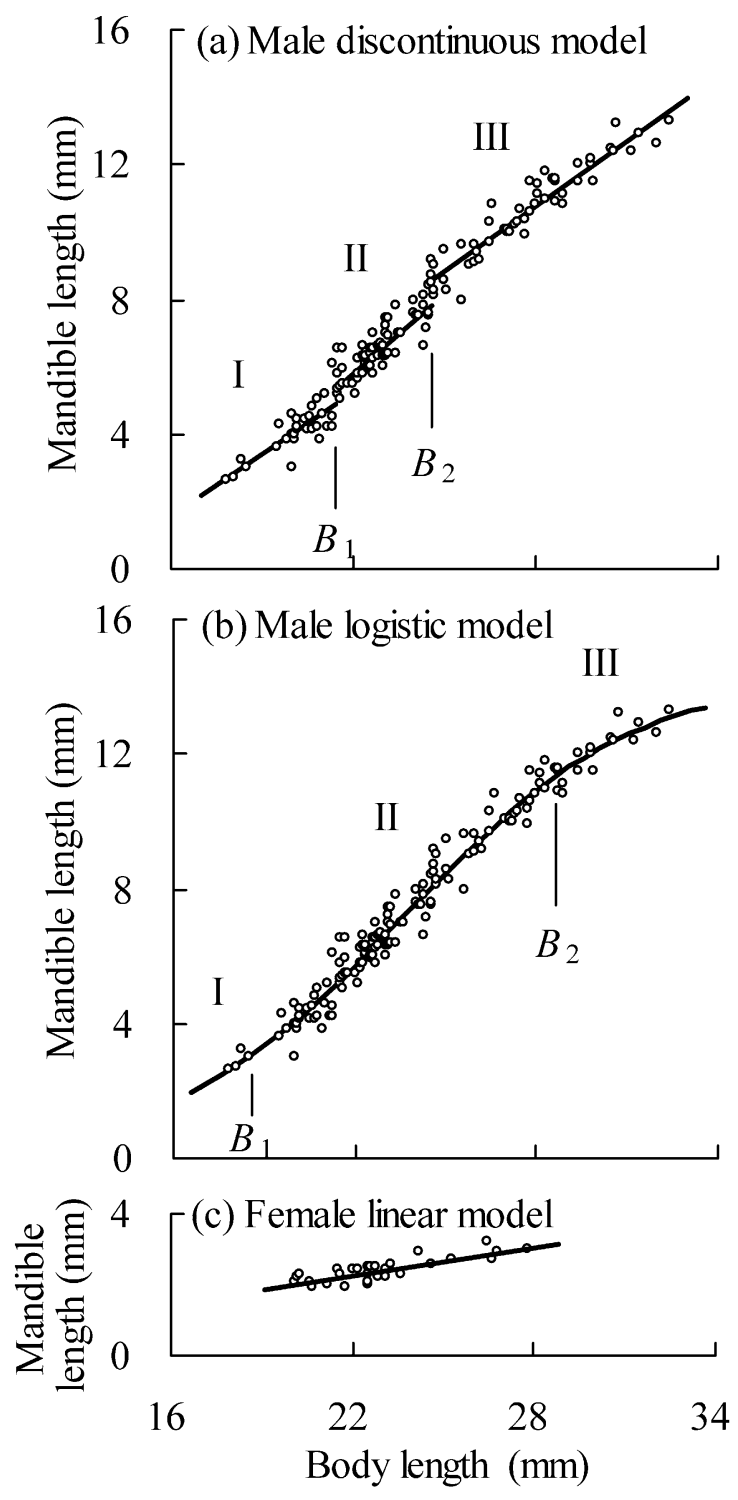

Fig. 3. (a) Discontinuous line with two break points fitted to the male data. I: $y=0.61 x-8.3$. II: $y=0.77 x-11.2$. III: $y=$ $0.64 x-7.2$. (b) Logistic curve fitted to the male data. $y=$ $14.3 /[1+489 \exp (-0.263 x)] . B_{1}$ and $B_{2}$ show the break points, at which the slope changes fastest. (c) Single line fitted to the female data. $y=0.13 x-0.62$.

model for the female data was a simple line (Fig. 3c) and no other model had substantial support (Table 2).

In the discontinuous linear model for the males (Fig. $3 \mathrm{a})$, the parameter $d$ was significantly different from zero at both break points, $B_{1}(t=3.03, \mathrm{df}=90, P=0.003)$ and $B_{2}(t=4.76, \mathrm{df}=115, P<0.0001)$, showing that the three regression lines were discontinuous.

Kappa statistic showed excellent agreement $(k=0.81, \mathrm{P}$ $<0.001$ ) between the classification of males on the basis of mandible shape and that using the discontinuous linear model (Table 3).

\section{DISCUSSION}

Beetles with dimorphic males in which intermediate sized males tend to be rare often exhibit sigmoidal scaling relationships between body length and horn or mandible 
TABLE 3. Comparison of the classification of males by mandible shape (Fig. 1) and using the discontinuous linear model (Fig. 3a).

\begin{tabular}{cccc}
\hline \multirow{2}{*}{ Mandible shape } & \multicolumn{3}{c}{ Discontinuous model } \\
\cline { 2 - 4 } & I & II & III \\
\hline No teeth & 26 & 4 & 0 \\
One pair of teeth & 3 & 61 & 11 \\
Two pairs of teeth & 0 & 0 & 43 \\
\hline
\end{tabular}

length (Eberhard \& Gutierrez, 1991; Kawano, 1995; Moczek et al., 2002; Emlen et al., 2005a). However, Rowland \& Emlen (2009) showed that beetles with trimorphic males, such as the dung beetle Oxysternon conspicillatum and stag beetle Odontolabis cuvera, in which intermediate-sized males are abundant, also exhibit sigmoidal scaling relationships.

Unlike in these beetles, there is not a clear sigmoidal scaling relationship between body length and mandible length in male $D$. rectus. Polynomial functions have often been used to detect dimorphism in beetles (Eberhard et al., 2000; Hongo, 2003; Tomkins et al., 2005; Harvey \& Gange, 2006). However, the results presented suggest that the fitting and comparison of a larger set of models may be a better way of detecting and distinguishing between monomorphism, dimorphism and polymorphism in scaling relationships. A subtle sigmoidal scaling relationship for male $D$. rectus, for which there is abundant data for intermediate-sized males, received some support in the statistical analyses carried out in this paper.

The best-fitting model for the males was a discontinuous line with two break points. Moreover, the classification of the males by the discontinuous linear model was in excellent agreement with that based on mandible shape. These results suggest that subtle allometric trimorphism possibly exists in male $D$. rectus. On the other hand, as expected, the best-fitting model for females was a linear model. This is not surprising, but there are very few studies providing such statistical evidence for a linear model. As shown in Fig. 3c, body length and mandible length varies little in females. Therefore, it is difficult to describe accurately the scaling relationship between them.

Many studies on dimorphic beetles have shown that there are behavioural differences between large and small males. For example, large males fight with other males for possession of females and food, whereas small males avoid fighting with other males and use other tactics (Eberhard, 1982; Goldsmith, 1987; Siva-Jothy, 1987; Rasmussen, 1994; Emlen, 1997; Moczek \& Emlen, 2000; Iguchi, 2001b; Hongo, 2003). On the other hand, Rowland \& Emlen (2009) suggest that male trimorphism may be related to three reproductive tactics; a dominant (fight and guard) tactic, a subordinate (sneak) tactic, and a female-mimicry tactic. Unfortunately, there are no detailed studies on the behaviour of males of $D$. rectus that could be used to evaluate this hypothesis.

Inukai (1924) examined the mandibles of the stag beetles Cladognathus inclinatus and Lucanus maculifemoratus and suggests that the female form is the primary one

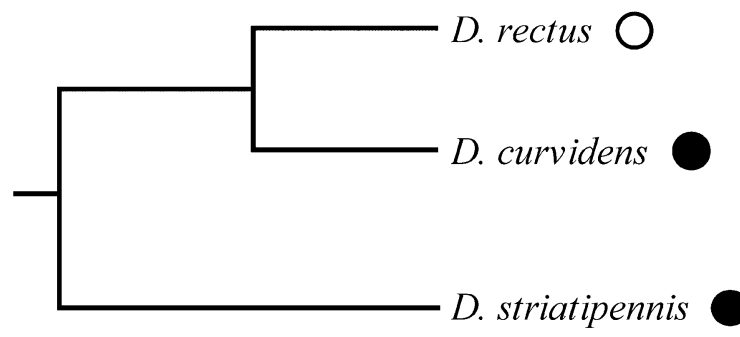

Fig. 4. Phylogenetic relationships between D. rectus, D. curvidens and $D$. striatipennis based on the mitochondrial DNA study of Hosoya et al. (2003). Small males of D. rectus have no distinct teeth on their mandibles (open circle), whereas small males of $D$. curvidens and D. striatipennis have one pair of distinct teeth on their mandibles (filled circles).

from which the male form was derived. Rowland \& Emlen (2009) also indicate that the smallest male forms in the beetle families Scarabaeidae, Lucanidae and Curculionidae resemble females. In $D$. rectus, however, the mandibles of females do not resemble those of small males but those of intermediate-sized males (Fig. 1).

Interestingly, even small males of $D$. striatipennis and $D$. curvidens, two species closely related to D. rectus, have, like females, one pair of distinct teeth on their mandibles (Kurosawa et al., 1988; Iguchi, pers. observ.). The phylogenetic relationships between these three species (Fig. 4; Hosoya et al., 2003) suggest that for small males the ancestral condition may have been one pair of teeth on the mandibles. Consequently, the trimorphism in $D$. rectus may have arisen from the loss of the teeth in small males. Several studies have shown that horned beetles repeatedly gained and lost their horns during evolution (Moczek, 2005; Emlen et al., 2005b, 2007). However, the evolution of the teeth on the mandibles of stag beetles remains to be explored.

\section{REFERENCES}

Burnham K.P. \& Anderson D.R. 2002: Model Selection and Multimodel Inference: A Practical Information-Theoretic Approach. 2nd ed. Springer, New York, 488 pp.

Clark J.T. 1977: Aspects of variation in the stag beetle Lucanus cervus (L.) (Coleoptera: Lucanidae). - Syst. Entomol. 2: 9-16.

Cook D. 1987: Sexual selection in dung beetles. I. A multivariate study of morphological variation in two species of Onthophagus (Scarabaeidae: Onthophagini). — Aust. J. Zool. 35: 123-132.

EBERHARD W.G. 1982: Beetle horn dimorphism: making the best of a bad lot. - Am. Nat. 119: 420-426.

Eberhard W.G. \& Gutierrez E.E. 1991: Male dimorphism in beetles and earwigs and the question of developmental constraints. - Evolution 45: 18-28.

Eberhard W.G., Garcia-C J.M. \& Lobo J. 2000: Size-specific defensive structures in a horned weevil confirm a classic battle plan: avoid fights with larger opponents. - Proc. $R$. Soc. Lond. (B) 267: 1129-1134.

EMLEN D.J. 1997: Alternative reproductive tactics and maledimorphism in the horned beetle Onthophagus acuminatus (Coleoptera: Scarabaeidae). - Behav. Ecol. Sociobiol. 41: 335-341. 
EMLEN D.J. \& NiJhout H.F. 2000: The development and evolution of exaggerated morphologies in insects. - Annu. Rev. Entomol. 45: 661-708.

EmLen D.J., Hunt J. \& Simmons L.W. 2005a: Evolution of sexual dimorphism and male dimorphism in the expression of beetle horns: phylogenetic evidence for modularity, evolutionary lability, and constraint. - Am. Nat. 166: S42-S68.

Emlen D.J., Marangelo J., Ball B. \& Cunningham C.W. 2005b: Diversity in the weapons of sexual selection: horn evolution in the beetle genus Onthophagus (Coleoptera: Scarabaeidae). - Evolution 59: 1060-1084.

Emlen D.J., Lavine L.C. \& Ewen-Campen B. 2007: On the origin and evolutionary diversification of beetle horns. Proc. Nat. Acad. Sci. USA (Suppl. 1) 104: 8661-8668.

FLEISS J.L. 1971: Measuring nominal scale agreement among many raters. - Psychol. Bull. 76: 378-382.

FLeISS J.L. 1981: Statistical Methods for Rates and Proportions. 2nd ed. Wiley, New York, $321 \mathrm{pp}$.

GoldSMIth S.K. 1985: Male dimorphism in Dendrobias mandibularis Audinet-Serville (Coleoptera: Cerambycidae). $-J$. Kans. Entomol. Soc. 58: 534-538.

GolDSMITH S.K. 1987: The mating system and alternative reproductive behaviors of Dendrobias mandibularis (Coleoptera: Cerambycidae). - Behav. Ecol. Sociobiol. 20: 111-115.

Harvey D.J. \& GANGE A.C. 2006: Size variation and mating success in the stag beetle, Lucanus cervus. - Physiol. Entomol. 31: 218-226.

Hongo Y. 2003: Appraising behaviour during male-male interaction in the Japanese horned beetle Trypoxylus dichotomus septentrionalis (Kono). - Behaviour 140: 501-517.

Hosoya T., Araya K. \& Shirota Y. 2003: Molecular phylogeny of Japanese stag beetles of the genes Dorcus (Coleoptera, Lucanidae) and its allied genera inferred from mitochondrial COI gene sequences. - Elytra 31: 127-142.

IGUCHI Y. 1992: Individual variation in males of Macrodorcas rectus (Motshulsky). - Kontyu to Shizen 27: 16-19 [in Japanese].

IGUCHI Y. 1998: Horn dimorphism of Allomyrina dichotoma septentrionalis (Coleoptera: Scarabaeidae) affected by larval nutrition. - Ann. Entomol. Soc. Am. 91: 845-847.

IGUCHI Y. 2001a: Male morphological dimorphism in the stag beetle, Dorcus rectus (Coleoptera: Lucanidae). - Spec. Publ. Jpn Coleopt. Soc. 1: 201-204.

IgUchI Y. 2001b: Differences in the frequency of fights between minor and major males in the horned beetle Trypoxylus dichotomus septentrionalis (Coleoptera: Scarabaeidae). Entomol. Rev. Japan 56: 11-14.

IGUCHI Y. 2002: Further evidence of male trimorphism in the horned beetle Trypoxylus dichotomus septentrionalis (Coleoptera, Scarabaeidae). - Spec. Bull. Jpn. Soc. Coleopterol. 5: 319-322.

INUKAI T. 1924: Statistical studies on the variation of stag beetles. - Trans. Sapporo Nat. Hist. Soc. 9: 77-91.
KAWANO K. 1995: Horn and wing allometry and male dimorphism in giant rhinoceros beetles (Coleoptera: Scarabaeidae) of tropical Asia and America. - Ann. Entomol. Soc. Am. 88: 92-99.

Kawano K. 1997: Cost of evolving exaggerated mandibles in stag beetles (Coleoptera: Lucanidae). - Ann. Entomol. Soc. Am. 90: 453-461.

KNELL R.J. 2009: On the analysis of non-linear allometries. Ecol. Entomol 34: 1-11.

Kurosawa Y., Okajima S. \& Yamaguchi S. 1988: An Illustrated Book of Japanese Stag Beetles. Hoikusha, Osaka, 207 pp. [in Japanese].

MoczeK A.P. 2005: The evolution and development of novel traits, or how beetles got their horns. - BioScience 55: 937-951.

Moczek A.P. \& EmLen D.J. 2000: Male horn dimorphism in the scarab beetle, Onthophagus taurus: do alternative reproductive tactics favor alternative phenotypes? - Anim. Behav. 59: 459-466.

Moczek A.P., Hunt J., Emlen D.J. \& Simmons L.W. 2002: Threshold evolution in exotic populations of a polyphenic beetle. - Evol. Ecol. Res. 4: 587-601.

Motulsky H. \& Christopoulos A. 2004: Fitting Models to Biological Data Using Linear and Nonlinear Regression: A Practical Guide to Curve Fitting. Oxford University Press, Oxford, $351 \mathrm{pp}$.

RASMUSSEN J.L. 1994: The influence of horn and body size on the reproductive behavior of the horned rainbow scarab beetle Phanaeus difformis (Coleoptera: Scarabaeidae). - J. Insect Behav. 7: 67-82.

Rowland J.M. \& EmLEn D.J. 2009: Two thresholds, three male forms result in facultative male trimorphism in beetles. Science 323: 773-776.

Shiokawa T. \& IWAhashi O. 2000: Mandible dimorphism in male of a stag beetle, Prosopocoilus dissimilis okinawanus (Coleoptera: Lucanidae). - Appl. Entomol. Zool. 35: 487-494.

Siva-Jothy M.T. 1987: Mate securing tactics and the cost of fighting in the Japanese horned beetle, Allomyrina dichotoma L. (Scarabaeidae). - J. Ethol. 5: 165-172.

Tatsuta H., Mizuta K. \& Aкімото S. 2001: Allometric patterns of heads and genitalia in the stag beetle Lucanus maculifemoratus (Coleoptera: Lucanidae). - Ann. Entomol. Soc. Am. 94: 462-466.

Tatsuta H., Mizuta K. \& Акімото S. 2004: Relationship between size and shape in the sexually dimorphic beetle Prosopocoilus inclinatus (Coleoptera: Lucanidae). - Biol. J. Linn. Soc. 81: 219-233.

Tomkins J.L., Котiaho J.S. \& LeBas N.R. 2005: Matters of scale: positive allometry and the evolution of male dimorphisms. - Am. Nat. 165: 389-402.

Received November 11, 2010; revised and accepted July 2, 2012 